\title{
Continuous Forecasting of Ship Sway Based on LSSVM
}

\author{
Shi Bo-Wen ${ }^{1, a}$ and Zhou Bo ${ }^{1, b}$ \\ ${ }^{1}$ Navigation College, Dalian Maritime University, Dalian 116026, China \\ azhang-xy08@163.com, ${ }^{b}$ liuzjj@online.In.cn
}

Keywords: Least square support vector machine, Ship sway, Continuous forecasting.

\begin{abstract}
Least square support vector machine (LSSVM) algorithm is suitable for the data processing based on finite number of training samples to forecast the unknown data by a nolinear model. It has preponderance for solving the small sample, nonlinearity problems. Without prior information of sea waves and the state equations of ship motions, only using the real measured roll and pitch data ,the LSSVM method is applied to solve the problem of short time series Forecasting. Results show that the method satisfies the need of online forecasting within 15 seconds, and continuous forecasting can be realized by sliding the window.
\end{abstract}

\section{Introduction}

The ships sway forecast is of great significance to the safety of carrier aircraft taking off and landing. According to safety requirement of aircraft taking off and landing on ship rolling, pitch and heave, we take method of very short-range forecast to find a time window in the coming time suitable for landing. Using this the commander can inform pilots of situations on ship sway when aircrafts plan for landing, and can help the commander make decision [1].

At present, related research institutes in the domestic academic circlesmainly includeHarbin Engineering University,Dalian Maritime University and 702 research institute of China Shipbuilding Industry Corporation [1]. Harbin Engineering University mainly makes forecasts to the swaying data of ship model tests, using methods including auto regressive method(ARM) [2], least square method [3], wave method, Kalman filtering method, cycle map method, projection pursuit method, neural network method [4] etc. While Dalian Maritime University mainly use chaotic time series analysis method [5], and 702 research institute mainly use ARMA method and neural network method. In the last decade, there is no new research production published. Overall, in the related materials we have consulted, there is no research production that can make effective forecasts to real ship test data for up to 15 seconds.

We take support vector machine method to make very short-range forecasts to real ship test data.

\section{Collection and Analysis of Ship Swaying Data}

Experimental Equipment Introduction. We use two kinds of equipment to measure movement posture of ship: one is membrane potential level sensor, the second is movement posture measurement products VM-i designed by Beijing company VMSENS.

Data measured by membrane potential level sensor can be collectedautomaticallyby data acquisition card transferred to PC via serial port. The output data structure is: serial number, roll, pitch. The output data structure of VMSENS VM-i is: yaw, pitch, roll, magnetic field strength of $\mathrm{x}$-axis, magnetic field strength of $\mathrm{y}$-axis, magnetic field strength of $\mathrm{z}$-axis, gyroscope output of $\mathrm{x}$-axis, gyroscope output of $y$-axis, gyroscope output of z-axis, acceleration of $x$-axis, acceleration of $y$-axis, acceleration of z-axis.

Condition of Environment. We collect swaying data of a ship and a training ship, using membrane potential level sensor and VM-i respectively. The first time we measured data of one ship from June 12th 2011 to June 17th 2011, in the Yellow Sea. The second time we measured data from August 2nd 2011 to August 12th 2011, when a training ship was on a visit overseas. This time the 
measurement area is from Vladivostok, Russia to Wonsan, South Korea and from Wonsanc to Lüshun, China.

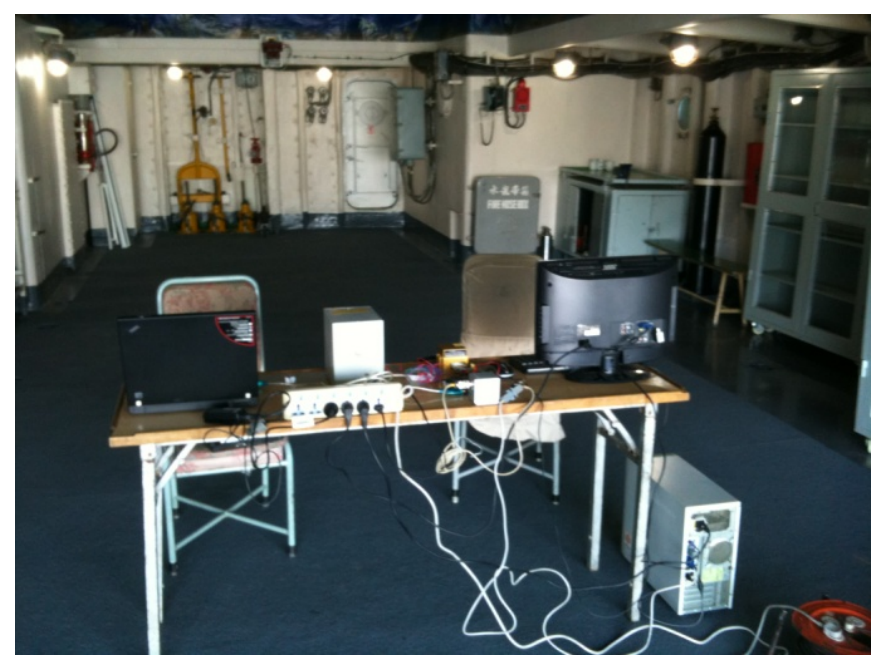

Fig. 1 Experiment site of movement posture measurement.

\section{Least Square Support Vector Machine}

Support vector machine(SVM) is a kind of machine learning algorithm proposed during the 90's of 20th century. In designing this algorithm, both minimizing of sample point error and minimizing of model structural risk is considered, so generalization of this model is guaranteed. As an extension of SVM, LSSVM has been applied in many fields, such as pattern recognition and time series modeling.

Modeling process of LSSVM can be considered as a nonlinear mapping $\varphi$ from low dimension space to higher dimension space. So LSSVM can transfer a nonlinear regression problem in low dimension space to a linear regression problem in higher dimension space. Let $X=\left\{x_{1}, x_{2}, \cdots, x_{n}\right\}$ be the input time series, to forecast it we need to map $\mathrm{X}$ onto higher dimension eigenspace $\mathrm{F}$ :

$$
f(X)=w \cdot \varphi(X)+b, \quad \varphi: R^{n} \rightarrow F
$$

$\mathrm{b}$ is domain value.

Optimization object of LSSVM is:

$$
\begin{aligned}
& \min _{w, b, e} J(w, e)=\frac{1}{2} w^{T} w+\frac{1}{2} \gamma \sum_{k=1}^{N} e_{k}^{2} \\
& \text { s.t. } \quad y_{k}=w^{T} \varphi\left(x_{k}\right)+b+e_{k}, k=1, \cdots, \quad \mathrm{N} .
\end{aligned}
$$

where $\varphi\left(x_{k}\right)$ is a map of $x_{k}$ in higher dimension space. Error $e_{k} \in \mathrm{R}$. Cost function $J(w, e)$ is structural risk, and $\gamma$ an adjustable constant.

By solving the equation above, we get a regression model:

$$
y(x)=\sum_{i=1}^{N} \alpha_{i} \phi\left(x_{i}\right)^{T} \phi\left(x_{j}\right)+b
$$

Let $\Omega_{i j}=\phi\left(x_{i}\right)^{T} \phi\left(x_{j}\right)=K\left(x_{i}, x_{j}\right)$ be a matrix of kernel function, we get:

$$
y(x)=\sum_{i=1}^{N} \alpha_{i} K\left(x, x_{i}\right)+b
$$

where $\alpha_{i}$ is lagrange multiplier, and choose RBF as kernel function $K\left(x, x_{i}\right)$, so: 


$$
K\left(x, x_{i}\right)=\exp \left\{-\frac{\left\|x-x_{i}\right\|^{2}}{2 \sigma^{2}}\right\}
$$

\section{Continuous Forecasting Method of Ship Swaying}

Continuous Forecasting Method Based on Single Step Iteration. Suppose the number of training samples is $m$. First we establish the mapping relations between samples $\left\{x_{1}, x_{2}, \cdots, x_{p}\right\}$ and $\left\{x_{p+1}\right\}$, then we pan the training window until the mapping relations $f: R^{p} \rightarrow R$ between $\left\{x_{m-1}, x_{m-2}, \cdots, x_{m-p}\right\}$ and the forecasting target $\left\{x_{m}\right\}$ is established, where $p$ is embedding dimension. We get input matrix and output matrix of training:

$$
X_{s v m}=\left[\begin{array}{cccc}
x_{1} & x_{2} & \cdots & x_{p} \\
x_{2} & x_{3} & \cdots & x_{p+1} \\
\vdots & \vdots & \ddots & \vdots \\
x_{m-p} & x_{m-p+1} & \cdots & x_{m-1}
\end{array}\right], Y_{s v m}=\left[\begin{array}{c}
x_{p+1} \\
x_{p+2} \\
\vdots \\
x_{m}
\end{array}\right]
$$

We can get nonlinear relationship $\varphi$ between $X_{s v m}$ and $Y_{s v m}$ by LSSVM training: $Y_{s v m}=\varphi X_{s v m}$.

The trained $\varphi$ can be used for forecasting. A detailed description of the steps follows.

(1) Choose a continuous sequence of length $\mathrm{m}$ from the sample sequence to be the training data.

(2) Using LSSVM method, as the cost function is:

$$
F_{p, L}\left(\gamma_{j}, \sigma_{j}^{2}\right)=\sum_{i=1}^{L} e_{i}^{2}=\sum_{i=1}^{L}\left(x_{m+i}-x_{m+i}^{*}\right)^{2}
$$

where, $F_{p, L}\left(\gamma_{j}, \sigma_{j}^{2}\right)$ is sum of error square between forecasting value and real value on each time points. $p$ is embedding dimension and $\mathrm{L}$ is step numbers of continuous forecasting.

(3) Train the LSSVM using the optimal solution $\left(\gamma_{\text {best }}, \sigma_{\text {best }}^{2}\right)$, and get $\left(a^{*}, b^{*}\right)$.

(4) Substitute $\left(a^{*}, b^{*}\right)$ in Eq. (4). Forecast the estimated value of $x_{m+1}$, using the training vector $X_{1}=\left\{x_{\mathrm{n}-\mathrm{p}+1}, x_{\mathrm{n}-\mathrm{p}+2}, \cdots, x_{m}\right\}$ as input. The estimated value of $x_{m+1}$ is $x_{m+1}^{*}$.

(5) Forecast the estimated value $x_{m+2}^{*}$ with the vector $X_{2}=\left\{x_{\mathrm{n}-\mathrm{p}+2}, \cdots, x_{m}, x^{*}{ }_{m+1}\right\}$ to be input.

(6) Likewise, we can get predicted value $x^{*}{ }_{m+L}$ after $L$ steps.

Multi-Step Continuous Forecasting Method. Using the single step method mentioned above, when $\mathrm{L}$ is bigger, we need a large amount of calculation. If we take some data on known time points, and forecast data of time points(more the one) in unknown region, this is called multi-step forecast. We suppose the number of known time points and unknown time points is $\mathrm{P}$ and $i$. First we construct mapping relationship between input and output. Suppose the length of training data is $\mathrm{m}$, we build a training data matrix $D_{\text {train }}$. We set the window's width $p+i$, first we construct mapping relationship between data $\left\{x_{1}, x_{2}, \cdots, x_{p}\right\}$ and $\left\{x_{p+1}, x_{p+2}, \cdots, x_{p+i}\right\}$, then we pan the training window until we construct mapping relationship between vector $\left\{x_{m-p+1}, x_{m-p+2}, \cdots, x_{m}\right\}$ and the forecasting target vector $\left\{x_{m+1}, x_{m+2}, \cdots, x_{m+i}\right\}$. Now we get input and output matrix of LSSVM.

$$
X_{s v m}=\left[\begin{array}{cccc}
x_{1} & x_{2} & \cdots & x_{p} \\
x_{2} & x_{3} & \cdots & x_{p+1} \\
\vdots & \vdots & \ddots & \vdots \\
x_{m-p-i+1} & x_{m-p-i+2} & \cdots & x_{m-i}
\end{array}\right] Y_{s v m}=\left[\begin{array}{cccc}
x_{p+1} & x_{p+2} & \cdots & x_{p+1} \\
x_{p+2} & x_{p+3} & \cdots & x_{p+\mathrm{i}+1} \\
& \vdots & & \\
& & & \\
x_{m-i+1} & x_{m-i+2} & \cdots & x_{m}
\end{array}\right]
$$

Similarly, we can get $Y_{s v m}=\varphi \cdot X_{s v m}$.

After training, we can use the mapping relationship $\varphi$ to do multi-step forecast. 


\section{Forecast to Real Ship Swaying Data}

Instruction on real ship swaying data. We choose two pieces of data for forecasting test from the measured data. The first piece is roll data of the 27th recording period in June 14th measured on board one ship. Through calculation, we get the significant value is 1.63 degrees. Fig. 2 is the roll spectrum and the main cycle is 7.89s.

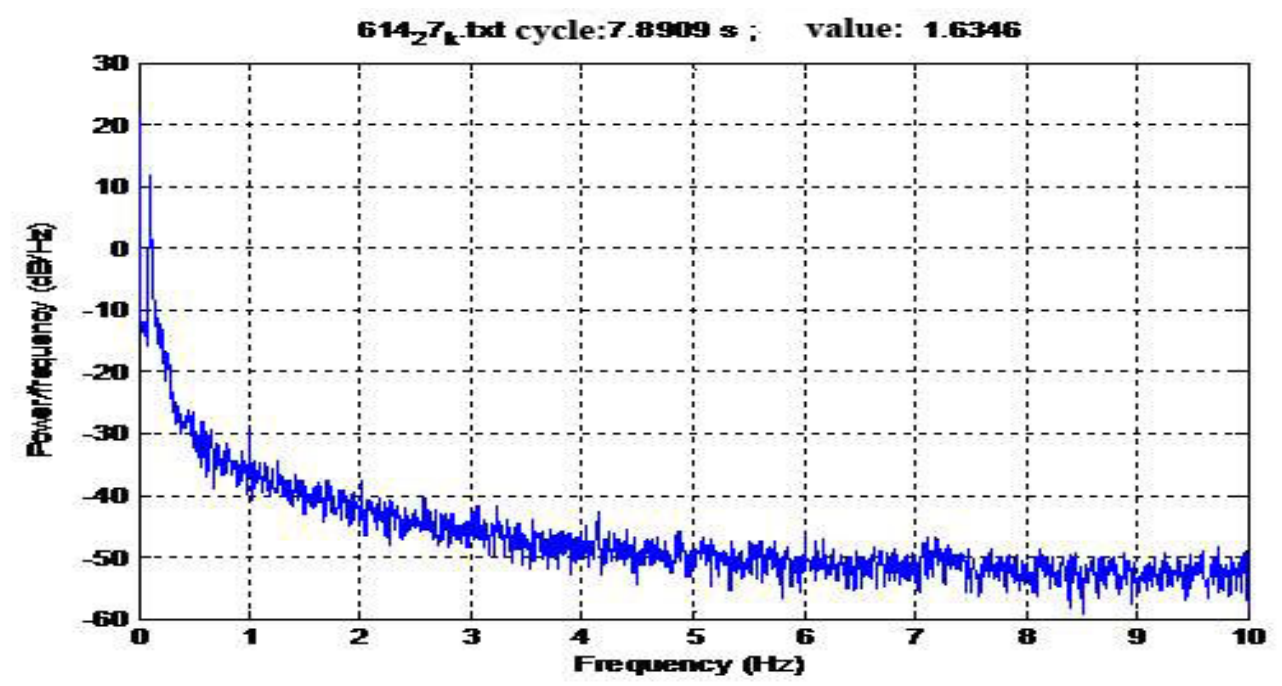

Fig. 2 Roll spectrum calculated from real ship data in June 14th.

The second piece is pitch data of a training ship sailing from Vladivostok, Russia to Wonsan, South Korea.Through calculation, we get the significant value is 2.56 degrees. Fig. 3 is the pitch spectrum and the main cycle is 5.11s.

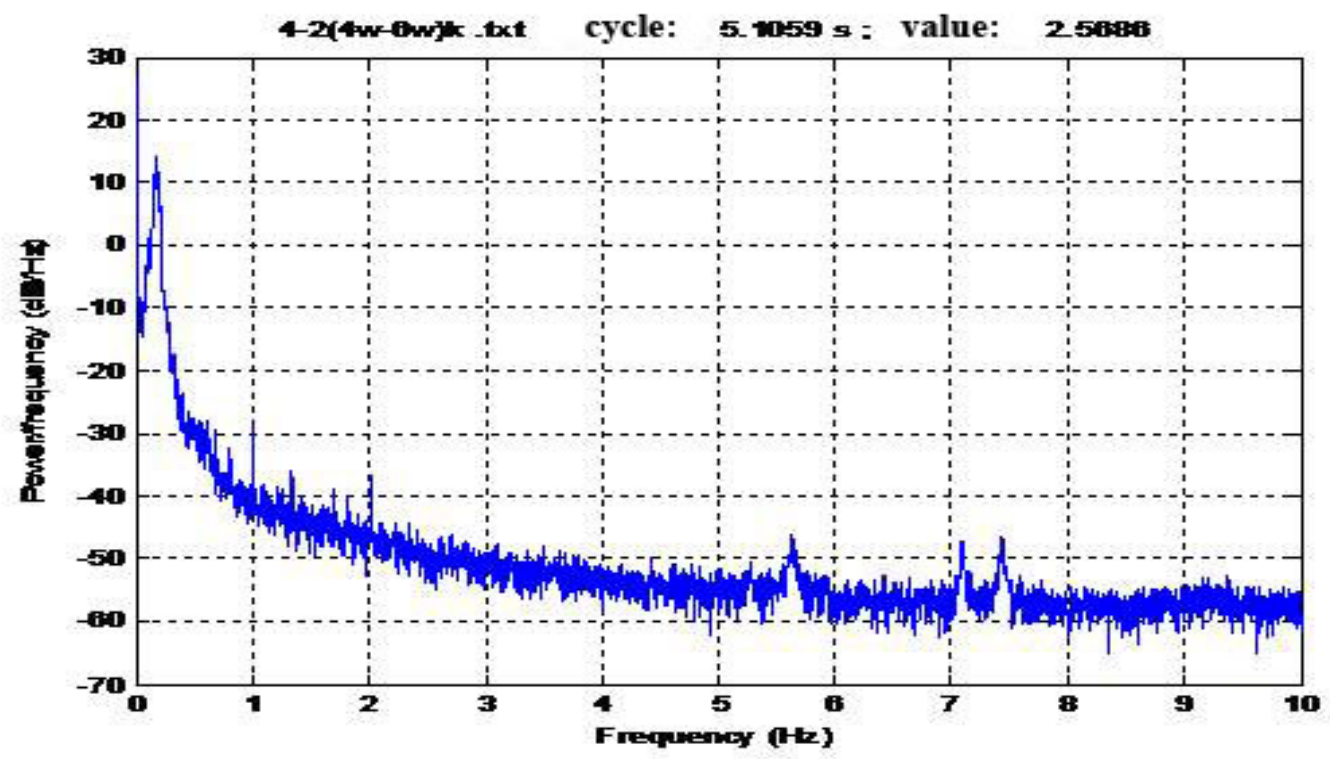

Fig. 3 Pitch spectrum calculated from real ship data.

Preprocessing of Real Ship Data. The fact that variables of input vector have too big difference will affect the training result, so the variables need normalization treatment. Suppose $x_{\max }$ and $x_{\min }$ is the maximum and minimum of the training set, $x$ is real swaying data and $\bar{x}$ is the normalized result, then:

$$
\bar{x}=\frac{x_{i}-x_{\min }}{x_{\max }-x_{\min }}
$$


In this way the training data is transferred to the range [0,1]. Finally we can use the equation follows to transfer it back to real swaying data:

$$
x_{i}=\left(x_{\max }-x_{\min }\right) \bar{x}+x_{\text {min }}
$$

By normalization treatment the ship swaying data will distribute in significant range.

\section{Analysis on Forecasting Result.}

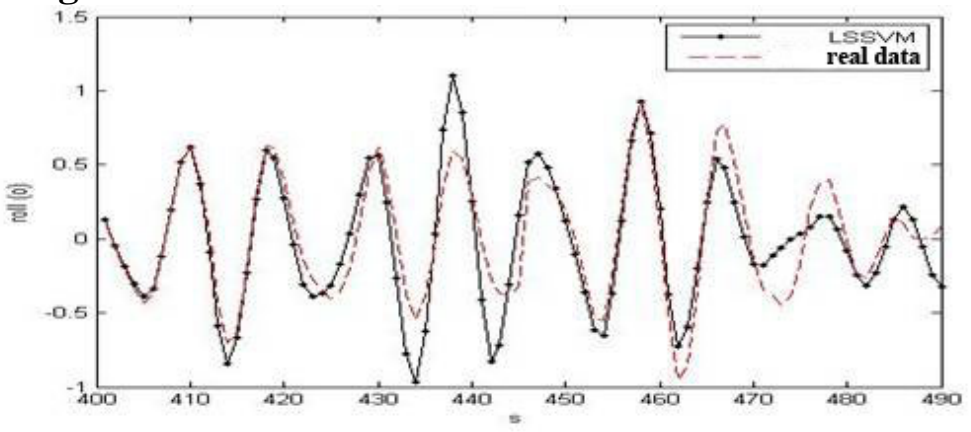

Fig. 4 Forecast to ship roll data (90 seconds altogether).

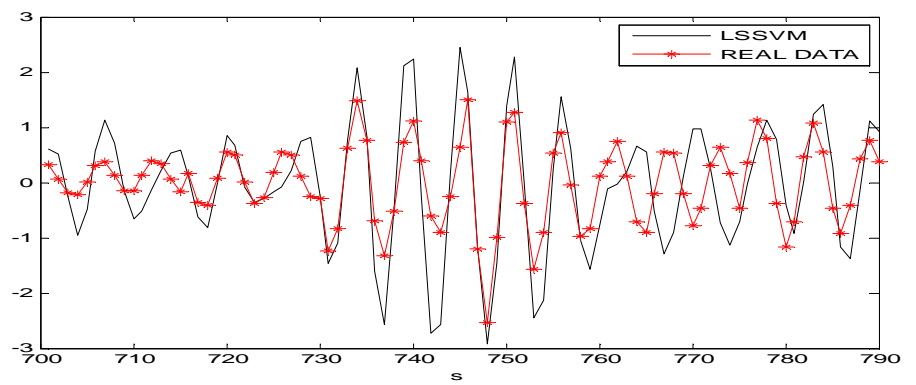

Fig. 5 Forecast to training ship pitch data (90 seconds altogether).

Evaluating Indicator. To evaluate forecasting accuracy of the model, we calculate error relative to significant value:

$$
\text { SMAPE }=\frac{\left|x_{m+i}-x_{m+i}^{*}\right|}{x_{1 / 3}}
$$

where $x_{m+i}$ is the real value, $x_{m+1}^{*}$ is the forecasting value, $m$ is the starting point of forecast and $\mathrm{i}$ is the forecasting target point. In the example above $i=1,2, \cdots, 15$ and $x_{1 / 3}$ is the significant value of real measured swaying data.

For comparison, in this experiment we make forecast to the data 15 seconds later each time, and we make forecast for 6 times continuously, totally 90s. We calculate average value of the error in 6 forecasts, and get the figure that error changes when the forecast length changes:

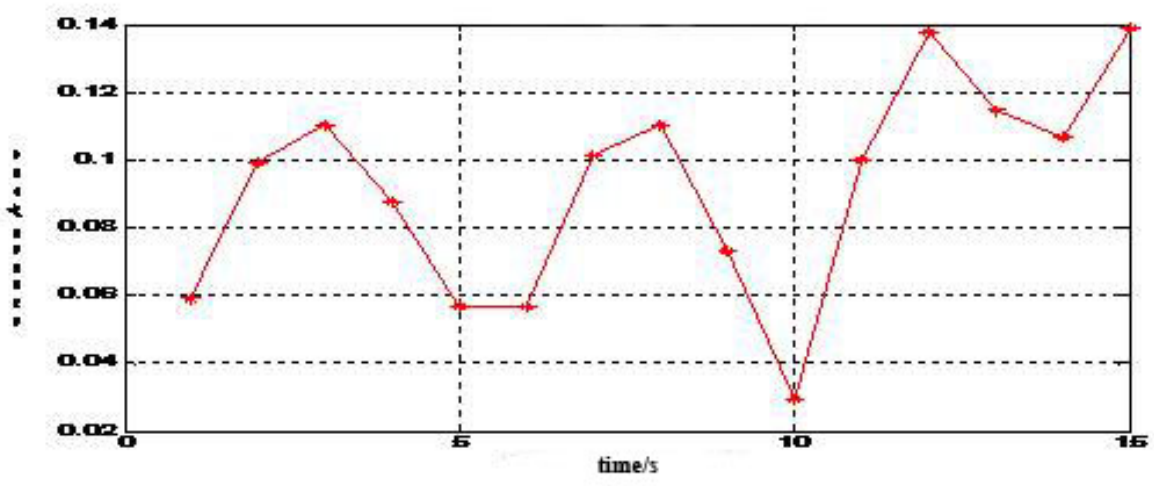

Fig. 6 Error of significant roll value in forecast process(15s). 


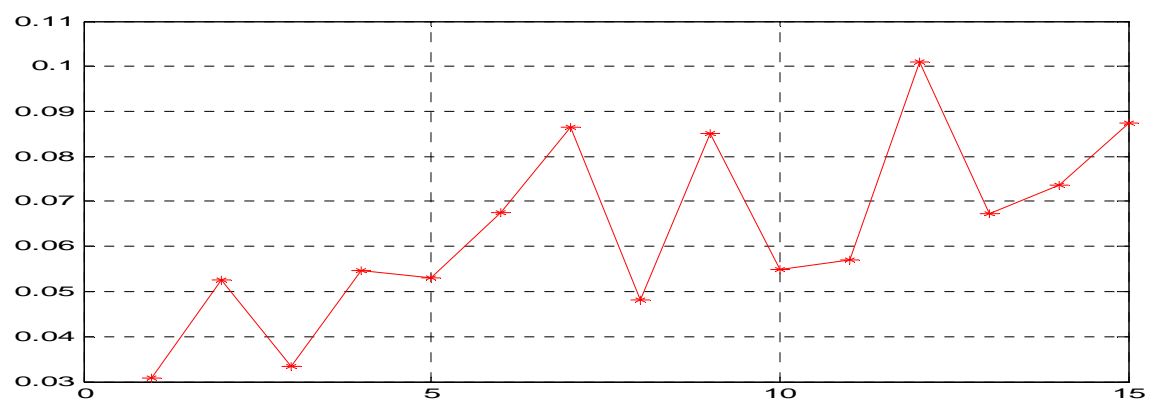

Fig. 7 Error of significant pitch value in forecast process(15s).

\section{Conclusion}

We build a single step continuous forecasting algorithm based on LSSVM, as LSSVM has big advantage in small sample modeling. This algorithm has quick calculation and can meet the demand of real-time operation. Furthermore, to solve the problem of error accumulation we propose multi-step algorithm. As multi-step algorithm give consideration to both training error and variety of swaying data, it has the advantages of small sample and strong generalization ability and is suitable for ship swaying online forecast.

Fig. 6 and Fig. 7 show that the relative error is lower than 12\% in 10s forecast and lower than 15\% in 15 s forecast. Therefore, in real ship swaying forecast the LSSVM method proposed here can be reliable for about 2 swaying cycle, which can meet the basic need of very short-range real ship forecast.

\section{References}

[1] S. B. Liu, P. Shi, J. H. Chen, Research on prediction of ship motion in wave. J. Guangzhou Marine Coll. 22(2) (2014) 21-23.

[2] X. Y. Peng, X. R. Zhao, N. X. Wei, A AR Algorithm of Large Ship Motion Forecast. Ship Eng. 5 (2001) 5-7.

[3] X. Y. Peng, C. D. Liu, Ship Motion Very Short Range Prediction based on Lattice Recursive Least Square Algorithm. Ship Mech. 16(1-2) (2012) 44-51.

[4] L. Wang, Research on Ship Motion Prediction Based on CMAC Neural Network. J. Math. Pract. Theory, 44(23) (2014) 0-165.

[5] B. Liu, F. Cai, A. G. Shi, Double Variables Local Linear Multi-step Prediction Methodon Ship Swaying Forecast. Navigation of China, 35(2) (2012) 33-38.

[6] Y. M. Chen, J. W. Ye, Test on Very Short-range Forecast on Ship Motion, Ship Sea Eng. 38(1) (2010) 13-20. 